\title{
Diacronie
}

Studi di Storia Contemporanea

$\mathrm{N}^{\circ} 21,1 \mid 2015$

Le città di Babele

\section{Firenze noir. Criminalità e marginalità a Firenze tra Otto e Novecento}

\section{Anna Pellegrino}

\section{(2) OpenEdition}

Journals

\section{Edizione digitale}

URL: http://journals.openedition.org/diacronie/1845

DOI: 10.4000/diacronie. 1845

ISSN: 2038-0925

\section{Editore}

Association culturelle Diacronie

\section{Notizia bibliografica digitale}

Anna Pellegrino, « Firenze noir. Criminalità e marginalità a Firenze tra Otto e Novecento », Diacronie [Online], Nº 21, 1 | 2015, documento 2, Messo online il 01 mars 2015, consultato il 19 avril 2019. URL http://journals.openedition.org/diacronie/1845; DOI : 10.4000/diacronie.1845 


\section{Diacronie}

N. 21 | 1|2015 Le città di Babele

\section{2/}

\section{Firenze noir. Criminalità e marginalità a Firenze tra Otto e Novecento}

Anna PELLEGRINO *

San Frediano, al cuore del grande e popoloso quartiere di Santo Spirito, tra la fine dell'Ottocento e l'inizio del '900 era il rione "nero" di Firenze. Nero in diversi sensi: in primo luogo, per la sua delinquenza endemica, direttamente connessa alla situazione sociale del quartiere, il più malfamato della città; in secondo luogo, perché oggetto privilegiato di una certa letteratura (ispirata ai Misteri di Parigi di Eugène Sue); in terzo luogo, perché estraneo ai movimenti socialisti e riformisti; nero, infine, perché nel 1901 un sesto della sua popolazione risultava ammonita o sorvegliata dalla polizia. Il mio saggio si propone di sondare una serie di fonti-per dipanare il rapporto fra il volto luminoso di Firenze, sede privilegiata del turismo colto europeo, e il "volto oscuro" "della marginalità, dell'esclusione, delle relazioni border line o oltre il confine della legalità», che si intrecciavano poche decine di metri più in là, separate solo dalla sottile striscia del fiume. 


\section{Il cuore nero di Firenze ${ }^{1}$}

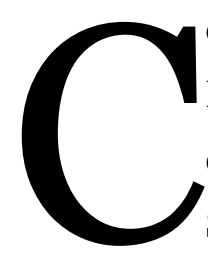

orreva voce che neppure la polizia osasse avventurarsi di notte in Borgo San Frediano. Anche i giornali ne parlavano come di un dato di fatto, quasi ovvio e scontato. San Frediano, al cuore del grande e popoloso quartiere di Santo Spirito, tra la fine dell'Ottocento e l'inizio del Novecento era il rione "nero" di Firenze.

Nero in diversi sensi. In primo luogo perché la cronaca nera trovava nel quartiere un facile alimento alle sue colonne. Assassinii, furti, ma più spesso liti e risse, testimonianze di una delinquenza endemica, non strutturata, direttamente connessa alla situazione sociale del quartiere, il più povero, il più disagiato, il più sordido $\mathrm{e}$ malfamato della città. Nero in secondo luogo perché era lo spazio privilegiato di una certa letteratura che ricalcava i Misteri di Parigi di Eugène Sue, impegnando alcuni degli scrittori fiorentini più noti, la cui fama talora era in grado di travalicare i confini cittadini, a raccontare storie dell'altra città, quella misteriosa e sotterranea dei rioni popolari. Nero in terzo luogo perché il rosso delle bandiere socialiste, che copriva gran parte dei quartieri cittadini e tutta la zona rossa delle periferie, si arrestava ai confini di San Frediano. Lì i socialisti non erano mai riusciti, fino a quell'epoca e per lungo tempo anche in seguito, a impiantare le loro strutture organizzative: quelle di mutuo soccorso, quelle cooperative e meno che mai una sezione del partito. Sventolavano invece spesso e volentieri in San Frediano le bandiere nere dell'anarchismo, o per dirlo in maniera meno enfatica, nelle bettole, nelle osterie, dai vinai di San Frediano si potevano trovare facilmente personaggi con la camicia nera e magari con un nastro o un fazzoletto rosso, che denotavano a quel tempo una sicura estrazione anarchica, una presenza largamente testimoniata anche dalle fonti di polizia e che rinviava al classico sovversivismo, in larga misura pre-partitico, dei ceti popolari dell'epoca. Nero, infine, perché al di là degli aspetti giornalistici o letterari, o semplicemente di "colore" politico, anche le nude cifre della statistica non lasciavano dubbi. Ugo Giusti, il capo dell'Ufficio di statistica del

\footnotetext{
${ }^{1}$ Questo saggio si propone di sondare una serie di fonti: letterarie, di polizia, inchieste mediche e statistiche, fonti giudiziarie e giornalistiche per analizzare il rapporto fra il volto luminoso della città dei fiori, sede privilegiata del turismo colto europeo, e il "volto oscuro" "della marginalità, dell'esclusione, delle relazioni border line o oltre il confine della legalità», che s'intrecciavano a poche decine di metri di distanza, separate solo dalla sottile striscia del fiume, che segnava il confine fra il centro storico e il quartiere popolare di San Frediano. Una versione assai ridotta di questo contributo è stata pubblicata negli atti del Convegno tenutosi a Catania dal 12 al 14 settembre 2013. ADORNO, Salvatore, CRISTINA, Giovanni, ROTONDO, Arianna (a cura di), Visibile/invisibile: percepire la città tra descrizioni e omissioni, Catania 12-14 settembre 2013, vol. I., Identità urbane: narrazioni, retoriche, rappresentazioni, Catania, Scrimm Edition, 2014, pp. 125-135.
} 
Comune di Firenze, studioso esperto e di valore riconosciuto, tanto da essere arrivato alla guida dell'Unione Statistica delle città italiane, in una indagine del suo ufficio dava delle cifre cupe e impressionanti. Al 1901, su 6.895 abitanti censiti nel rione di San Frediano, (comprese quindi donne, vecchi e bambini) ben 1.030 erano ammoniti o sorvegliati dalla polizia.

Che su queste basi trovasse alimento una letteratura romanzesca popolare e quasi noir che tentava di dipingere la piccola e un po' provinciale Firenze e i suoi quartieri popolari e centrali con gli stessi colori e le stesse intonazioni del ventre di Parigi, si può quindi spiegare. Anche le penne che vi si impegnarono non erano di trascurabile peso: da Giulio Piccini, a Pietro Ferrigni, a Carlo Lorenzini: scrittori per la verità tutti più noti, specie l'ultimo, con i loro rispettivi nomi d'arte, e cioè rispettivamente Jarro, Yorick, e Collodi².

Allo storico di oggi non può non venire il dubbio che queste autorevoli penne si fossero mosse non solo per l'evidenza dello stato di degrado delle zone "pericolose" della città, o per desiderio d'emulare i più noti esempi letterari parigini, ma anche $\mathrm{e}$ soprattutto perché su alcune di queste zone da loro descritte, prima sul ghetto e poi su San Frediano, si erano appuntate le curiosità dell'opinione pubblica, a sua volta sollecitata dalle mire delle classi dirigenti e degli speculatori desiderosi di ristrutturare quelle zone centralissime. Operazione riuscita nel caso del ghetto e anche - più tardi e parzialmente - nel caso di Santa $\mathrm{Croce}^{3}$, ma che non riuscì invece nel caso di San Frediano.

\section{L’invisibilità del nero}

Il nero, assorbendo tutta la luce, è invisibile; quello che veramente vediamo sono i riflessi della luce sugli oggetti di colore nero, riflessi che a volte mandano vividi lampi, altre restituiscono diverse tonalità di grigio.

Pressappoco la stessa cosa succedeva ai quartieri popolari di Firenze. Il vero quartiere nero, quello dei Camaldoli di San Lorenzo, è perso quasi del tutto nel buio

\footnotetext{
2 Fra gli altri: INVERNIZIO, Carolina, L'orfana del Ghetto: Romanzo storico sociale, Firenze, Salani, 1887; JARRO [PICCINI, Giulio], Firenze sotterranea, Firenze, Bemporad, 1900 (quarta ed., da cui si cita; la prima ed. è del 1884); ID, L'assassinio nel vicolo della luna, Milano, Fratelli Treves Tip. Ed., 1883; ID., La polizia del diavolo, Milano, Fratelli Treves, 1886; ID., I ladri di cadaveri, Reggio Emilia, Alberti, 2004 [1884]; COLLODI, Carlo [LORENZINI, Carlo], I misteri di Firenze. Scene sociali, Firenze, Fioretti, 1857; YORIK, [FERRIGNI, Pietro], Su e giù per Firenze, Firenze, Barbera, 1878.

3 Si veda a proposito: FEI, Silvano, Le vicende urbanistiche del quartiere di Santa Croce dalle origini ai nostri giorni, Firenze, Comune di Firenze assessorato all'urbanistica, 1986.
} 
della memoria. Ne sappiamo pochissimo: solo che era in condizioni incredibilmente degradate, tali da suscitare vergogna, ed evidentemente rimozione. Era stato il primo a sparire, rimosso fisicamente subito dopo la proclamazione di Firenze Capitale, e non ne era restato quasi niente nella memoria, se non una certa reputazione di pericolosità sociale dei suoi popolani: il Dolfi, il capopolo del 1859, proprio da quel rione proveniva 4 .

Un po' più visibile appare il secondo e il principale dei quartieri oscuri di Firenze, il quartiere del Ghetto. Non che fosse facile vedere dentro i suoi confini, che racchiudevano quello che era il vero centro geografico della città di Firenze, il complesso di isolati che dal Palazzo dell'Arcivescovado, ai margini della piazza del Duomo, arrivava toccando e quasi inglobando Palazzo Strozzi fino ai margini di Piazza della Signoria. Era una zona tanto privilegiata come collocazione spaziale quanto degradata come reale stato fisico. Sicuramente, in una specie di "domino" del degrado edilizio urbano, aveva influito la demolizione dei Camaldoli di San Lorenzo, che aveva spinto molti dei poverissimi abitanti espulsi da quella zona a rifugiarsi nel vicinissimo Ghetto. Lo stesso trasferimento della capitale a Firenze aveva provocato un singolare effetto di degrado a catena: la ricerca spasmodica di alloggi per i nuovi arrivati della burocrazia e i ceti dirigenti del nuovo Stato aveva finito con il provocare un fortissimo incremento dei prezzi delle case, espellendo i vecchi abitanti verso zone sempre peggiori e in condizioni di crescente sovraffollamento; inoltre, non potendosi costruire in tempi brevissimi tutti gli alloggi occorrenti, si era ricorsi ad uno straordinario incremento delle sopraedificazioni, ampliamenti e aggiunte al patrimonio edilizio esistente, che nelle zone più interne e centrali aveva provocato quasi automaticamente un drastico decremento della qualità e della salubrità degli ambienti5.

Tutto questo si era visto e si vedeva poco, dato che tutta l'attenzione era mirata verso l'opera di ristrutturazione urbanistica del Poggi e sulla costruzione del vasto e arioso anello dei viali e del piazzale Michelangelo ${ }^{6}$. Terminata rapidamente la breve

$4 \mathrm{Si}$ veda a proposito: SALVESTRINI, Arnaldo, "Giuseppe Dolfi: un capopopolo nella rivoluzione dei signori», in Rassegna Storica Toscana, 2/1969, pp. 221-232.

5 REDI, Paolo, Espansione e speculazione edilizia in Firenze Capitale, in La Toscana nell'Italia Unita. Aspetti e momenti di storia toscana, 1861-1945, Firenze, URPT, 1962, p. 464.

${ }^{6} \mathrm{Si}$ veda in generale sul piano urbanistico del Poggi: POGGI, Giuseppe, Sui lavori per l'ingrandimento di Firenze, Firenze, Barbera, 1882; DETTI, Tommaso, Firenze scomparsa, Firenze, Vallecchi, 1970; per un esame dettagliato dell'intera operazione si vedano FEI, Silvano, Nascita e sviluppo di Firenze città borghese, Firenze, G.\& G. Editrice, 1971; ed il più recente lavoro di CRESTI, Carlo, Firenze, capitale mancata, Architettura e città dal piano Poggi a oggi, Firenze, Electa, 1995. Su un piano più prettamente politico-economico si veda COPPINI, Romano Paolo, L'opera politica di Cambray-Digny sindaco di Firenze Capitale e ministro delle Finanze, Roma, Edizioni di storia e letteratura, 1975; una suggestiva e descrittiva analisi sulle 
avventura di Firenze capitale, cosa che aveva portato addirittura al fallimento del Comune di Firenze - il che certo non aveva facilitato l'opera di manutenzione del patrimonio edilizio nelle zone più povere - il problema del Ghetto assunse contorni e dimensioni che era sempre più difficile ignorare.

Si trattava però di contorni che venivano definiti non con le linee chiare e nette di una indagine statistica o sociale, ma seguendo le vie della letteratura o del bozzetto giornalistico e di colore. Carolina Invernizio, che era per l'appunto figlia di una di quelle famiglie di notabili e funzionari piemontesi arrivati a Firenze nel 1865, nel 1887 pubblicò un fortunato romanzo, dal titolo L'orfana del Ghetto, ambientato proprio nel vecchio centro di Firenze. Analogo successo ebbe una serie di articoli pubblicati da Giulio Piccini (Jarro) su «La Nazione» di Firenze e raccolti in volume sotto il titolo di Firenze sotterranea. A questa dimensione latomica e segreta alludevano anche volumi come quello di Carlo Lorenzini (Collodi), I misteri di Firenze, di cui uscì (1857) quello che doveva essere il primo volume, con un sottotitolo "scene sociali"; ma che poi non ebbe seguito; mentre il titolo fu poi ripreso diversi anni più tardi da Egisto Maccanti nel 1884. Sarebbe una impresa troppo ardua seguire in questa sede tutta la serie di romanzi e racconti, fra mistery e poliziesco ante litteram, che furono ambientati nel cuore di Firenze. Questa vasta produzione giornalistica e letteraria tendeva naturalmente a dare un quadro molto impressionista, con notevoli giochi di luci e ombre, delle condizioni del Ghetto.

Tuttavia era pur sempre un modo di rendere visibile una realtà, che paradossalmente proprio agli occhi più chiari e razionali degli indagatori sociali, degli ingegneri, dei medici, e dei rappresentanti delle istituzioni che componevano la Commissione d'indagine sulle condizioni abitative dell'isolato del Ghetto nominata dal Comune nel 1877, risultava in realtà assai difficile da esaminare, anzi proprio da vedere ${ }^{7}$.

Come recitava, infatti, la relazione della commissione stessa, nel 1881 , era stato assai difficile riuscire a compiere una indagine conoscitiva.

Non devesi tacere che quattro anni fa la cessata Amministrazione Comunale,
preoccupandosi dello stato e della condizione di alcune abitazioni meno salubri
della nostra città, $[. .$.$] nominò una commissione di cittadini della quale tutti noi ad$
eccezione di uno solo facemmo parte. È noto però che una prima

condizioni igieniche della città al tempo della Capitale ci è offerta dal cronista contemporaneo Camerani. CAMERANI, Sergio, Cronache di Firenze Capitale, Firenze, Olschki, 1971.

7 Cfr. la relazione della commissione pubblicata in FEI, Silvano, Firenze 1881-1898: la grande operazione urbanistica, Roma, Officina edizioni, 1977, pp. 240-253. 
sottocommissione, la quale ebbe ad occuparsi in special modo del quartiere del Ghetto, rimanendo come spaventata dalla vista di tanto luridume, declinò sollecitamente il mandato; e che una seconda sottocommissione nominata posteriormente si arrestò quasi sgomenta a mezza via, colpita dalla medesima impressione, se non declinò il mandato, certo non lo adempì̀ ${ }^{8}$.

Cosa avevano incontrato gli ingegneri e i medici del Comune, di tanto insostenibile alla vista, di così "spaventoso" da indurli a rinunciare più volte al proprio mandato di ispezione e di conoscenza? Seguiamoli nella prima visita che finalmente nel 1881 portarono a compimento:

La nostra prima visita incominciò dal quartiere di n. 72. Attraversammo l'immonda Piazza del Macello, e là pervenimmo salendo una stretta e ripida scala. Tre stanzucce, basse quanto appena l'ordinaria altezza di un uomo prendono poca luce dalla Corte già del Piazza. Gl'inquilini, per respirare un non buono, ma almeno più aperto aere stanno quasi tutto il giorno sulla porta terrena d'ingresso [...] a contatto delle immondezze del suolo. Non un vetro alle finestre. L'umidità e l'untume rivestono di uno strato permanente i mal connessi impiantiti [...] 9 .

Poco oltre, al numero 99,

trovammo una stanza affittata a Z.G. dove sopra un nudo saccone dormono cinque persone ricoperte appena dagli stessi miseri indumenti coi quali alla meglio si riparano durante il giorno. Sulle pareti di quella stanza si vede eloquentemente l'impronta di una lotta sostenuta con accanimento dagli abitatori contro immondi insetti notturni (cimex lectularius) ${ }^{10}$.

Il degrado fisico e quello igienico rischiavano poi di estendersi in degrado morale: gli appartamenti del Ghetto

hanno fatta una impressione penosa nella Commissione a causa del soverchio agglomeramento di individui che ivi si nota in una medesima stanza, in un medesimo letto, e, quel che ancora più rattrista, dell'uno e dell'altro sesso.

\footnotetext{
8 Ibidem, p. 240.

9 Ibidem, p. 242.

${ }^{10}$ Ibidem, p. 244.
} 
Non erano solo le abitazioni popolari a essere così degradate; vi erano dei servizi pubblici, ma non in condizioni molto migliori:

Il quartiere n. 82 destinato ad Albergo potrebbe in casi ordinari servire senza pericolo a una limitata famiglia; ma quando l'interesse di una industria privata spinge a collocare trenta letti, e i più a due posti, in quattro stanze, perché il provento ricavabile da quei promiscui e nomadi abitatori notturni è limitatissimo (centesimi venti per persona) qualunque ambiente non può non diventare insalubre ${ }^{11}$.

Non sappiamo bene chi fossero davvero quei «promiscui e nomadi abitatori notturni» ma certamente, il fatto che l'Albergo avesse «il grave inconveniente della doppia porta d'ingresso da due strade diverse» non doveva risultare loro sgradito, anzi, insinuava la commissione, probabilmente i clienti «si avvantaggiavano assai da questo comodo».

Questo aspetto, della pericolosità sociale che si collegava al degrado abitativo, era però soprattutto presente nel terzo caso che qui prendiamo in esame, e cioè nel quartiere di San Frediano, Oltrarno.

\section{3. "Il quartiere de' ladri, de' malviventi, Oltrarno"}

Protetto in qualche modo dalla trista fama dei Camaldoli prima e del Ghetto poi, San Frediano era vissuto nell'ombra; vicinissimo al centro, ma separato dal fiume, era rimasto un po' ai margini della considerazione dell'opinione pubblica, ma ora, all'inizio del XX secolo, tornava in primo piano, perché le zone più centrali della città erano ormai risanate.

Negli ultimi anni del XIX secolo il Ghetto, infatti, era stato «da secolare squallore a vita nova restituito», come recita la lapide dettata da Isidoro del Lungo che ancor oggi campeggia su Piazza della Repubblica, la piazza più elegante della città, ricavata appunto dall'abbattimento del Ghetto ${ }^{12}$.

\footnotetext{
${ }^{11}$ Ibidem, p. 242.

${ }_{12}$ Sulle ristrutturazioni urbanistiche degli anni Ottanta dell'Ottocento che portarono al risanamento del mercato vecchio, alla distruzione del ghetto e alla nascita dei nuovi mercati si veda CRESTI, Carlo, FEI, Silvano, «Le vicende del "risanamento" di Mercato Vecchio", in Storia Urbana, I, 1/1977, pp. 99-126; ID., Firenze 1881-1898: la grande operazione urbanistica, Roma, Officina Edizioni, 1977; Si veda inoltre FANELLI, Giovanni, Firenze architettura e città, Firenze, Vallecchi, 1973 e il più recente lavoro di FEI, Silvano, GOBBI SICA, Grazia, SICA, Paolo, Firenze, profilo di storia urbana, Firenze, Alinea, 1995, in particolare le pagine 127-152.
} 
In quella circostanza il Ghetto aveva finalmente avuto l'onore di una visibilità diffusa: una volta espulsi i poverissimi abitanti (che nell'occasione si erano potuti finalmente contare, vedendo quindi che erano un buon $20 \%$ in più di quelli che risultavano dai censimenti), espropriate le case, prima di por mano al piccone risanatore, era stata compiuta una campagna fotografica sistematica di ciò che si andava ad abbattere, e che comprendeva notevoli elementi di forte valore storico artistico. Vi si era tenuta perfino una festa in costume, che aveva visto le dame e i signori della buona società fiorentina aggirarsi per un'ultima rappresentazione che aveva per scenario lo spazio ancora occupato da quelle catapecchie ormai vuote, che al tempo erano sembrate troppo orride a vedersi ai tecnici del Comune, ma che ora si apprestava a diventare il luogo privilegiato di ritrovo dei ceti agiati, cosmopoliti e intellettuali che Firenze sempre più attraeva dall'Europa e dal mondo ${ }^{13}$.

Ora, quindi l'attenzione dell'opinione pubblica si spostava, alla ricerca del cuore nero di Firenze, verso quello che era stato da sempre il quartiere più popolare della città, un quartiere, "men noto del Ghetto perché più lontano dal Centro", ma che costituiva un problema anche maggiore, se non altro perché "del Ghetto assai più esteso". Si trattava di San Frediano, un borgo dove la pericolosità sociale era più alta che in ogni altra zona della città:

C'è di là d'Arno un quartiere dove i regolamenti municipali sono lettera morta [...] un quartiere dove la Polizia non va, a fare certe operazioni, se non a squadre di dodici o quattordici uomini; un quartiere dove il minimo subbuglio può tirar sulle strade, accalcare insieme a un tratto centinaia d'uomini e donne furenti! Vi dico che c'è un gruppo di strade segregate, che non servono come arterie di circolazione, ma sono tutte chiuse in sé e vi pullulano i ladri, i manutengoli: vi brulica la marmaglia, la bordaglia, la schiuma, il marame della popolazione, insieme accozzato.

Ł̀ interessante notare che in parte la stampa e gli osservatori contemporanei attribuivano questa pericolosità sociale del quartiere, che poi si esprimeva nelle cifre impressionanti della statistica criminale che abbiamo ricordato all'inizio, alla sua particolare conformazione urbanistica.

${ }_{13}$ Per inquadrare il fenomeno, cfr. PEMBLE, John, The Mediterranean Passion: Victorians and Edwardians in the South, Oxford, Faber \& Faber, 1987; e l'assai approfondito (anche se basato soprattutto su fonti letterarie) BUZARD, James, European Tourism, Literature, and the Ways to Culture, 180o-1918, Oxford, Oxford University Press, 1993; per la parte propriamente fiorentina e toscana, cfr. BOSSI, Maurizio, SEIDEL, Max (a cura di), Viaggio di Toscana. Percorsi e motivi del secolo XIX, Venezia, Marsilio, 1998. 
Si trattava, infatti, di un quartiere che presentava una variante interessante della forma urbana della città nel momento di transizione dagli assetti medievali e moderni a quelli dell'epoca contemporanea ${ }^{14}$.

Nonostante le trasformazioni subite nel periodo della Capitale, Firenze aveva conservato in buona parte il suo impianto medievale, senza una moderna divisione funzionale fra le varie zone della città. Come osservava il capo dell'Ufficio statistico del Comune, nel centro di Firenze accanto alle case torri e poi ai palazzi dei nobili e dei borghesi ricchi e influenti, sorgevano le casupole dei clientes, del personale di servizio, dei lavoratori poveri, che a volte si addensavano in zone o strade specifiche (le cui eco si risentono ancora in toponimi come via de' Cimatori, via degli Speziali, ecc.) spesso assai degradate:

L'omogeneità di carattere delle varie zone deve sempre intendersi in modo relativo: da noi assai meno che altrove esistono località abitate esclusivamente da alcune classi di popolazione: nelle zone più povere $[. .$.$] sono pure strade fiancheggiate da$ antichi palazzi [...] spesso a poca distanza da vie strette e affollate di popolazione povera ${ }^{15}$.

In San Frediano questi caratteri erano presenti, ma con una accentuazione degli aspetti di povertà del tessuto edilizio, che predisponevano ad una situazione di degrado igienico. Infatti, la zona d'Oltrarno, naturalmente meno pregiata del centro storico oltre il fiume, era stata caratterizzata dalla presenza di terreni appartenenti a chiese, conventi ed opere pie che avevano concesso a livello piccoli appezzamenti edificabili a artigiani e popolani, costituendo estese zone e intere vie con insediamenti abitativi popolari, con bassissimi standard di qualità già all'inizio, e poi ulteriormente peggiorate da una lunga storia di sovraedificazioni, aggiunte, modifiche e ristrutturazioni. Come osservava uno degli “ingeneri sociali” interessati all'opera di ristrutturazione:

tali casette sempre modeste, ma talora modestissime, si presentano nelle peggiori condizioni possibili riguardo alla loro disposizione interna avente sovente stanze una di seguito all'altra e male illuminate ${ }^{16}$.

\footnotetext{
14 In generale sull'argomento si veda: PINOL, Jean Luc, Le monde des villes au XIX siècle, Paris, Hachette, 1991.

${ }^{15}$ JARRO, Firenze sotterranea, cit., p. 47.

16 BELLINCIONI, Giovanni, Il problema del risanamento del quartiere di S. Frediano, Firenze, tip. G. Ramella, 1916, p. 19.
} 
Questo carattere artigiano e popolare del quartiere e questa estensione di un tessuto edilizio abitativo con standard qualitativi assai modesti fin dalle origini, realizzava un ambiente particolare, relativamente omogeneo, che permetteva agli abitanti di sfuggire ai meccanismi abituali di controllo, più facili dove il tessuto urbano era più regolare ed organizzato $^{17}$. Tutto ciò realizzava condizioni particolarmente favorevoli a chi voleva eludere la legge ${ }^{18}$ :

Dovete sapere che ogni casetta comunica con l'altra: da un orto si scavalla nell'altro: ci sono ad ogni piè sospinto passaggi e nascondigli [...] si va per cinque 0 sei strade, da una parte all'altra, scorrazzando nell'interno. Non basta. I tetti sono famigliari ai malviventi: vi camminano come voi pel vostro salotto : si vedono sempre scale preparate: ci è sempre gente che sa di dover scappare e altra che le tiene di mano [...] Il quartiere di cui parlo è così esteso e propizio ai malviventi, per l'agio che porge loro di balzare in pochi secondi da un punto estremo all'altro, che un contumace può rimanervi incolume eziandio per settimane, e di là spiccare il volo e mettersi in salvo ${ }^{19}$.

I rappresentanti della legge incontravano non poche difficoltà in questo ambiente:

La Polizia è brava, ma al Malborghetto, alla Sacra, al Campuccio, lungo le mura di San Rocco, in quel metodo di inseguimento nulla o poco può: a molti agenti insieme non è dato di saltar muri, terrazzi, né sapere in quel laberinto [...] la direzione che ha preso il furfante. Un agente solo non può arrischiarvisi, perché i tristi sono aiutati da innumerevoli compagni, da manutengoli; e l'agente, è reso in qualche cupa strozzatoia, assalito magari da cinque o sei persone, o a tradimento, è esposto a lasciarvi la vita ${ }^{20}$.

In queste condizioni era inevitabile che San Frediano (che fra l'altro era stata la zona in cui si era rifugiata in gran parte la popolazione povera espulsa dal Ghetto) diventasse il luogo

\footnotetext{
${ }_{17}$ Sul carattere artigiano della città mi permetto di rimandare al mio PELLEGRINO, Anna, $L a$ città più artigiana d'Italia. Firenze 1861-1929, Milano, Franco Angeli, 2012.

${ }^{18}$ Sul caso italiano in generale si veda DAVIS, John, Legge e ordine. Autorità e conflitti dal 1790 al 1900, Milano, Franco Angeli, 1989; cfr. ora sul caso parigino DELUERMOZ, Quentin, Policiers dans la ville. La construction d'un ordre public à Paris (1854-1914), Paris, Publications de la Sorbonne, 2012.

${ }^{19}$ JARRO, Firenze sotterranea, cit., p. 41.

${ }^{20}$ Ibidem.
} 
dove vivono i peggiori arnesi; uomini che hanno trascorso la vita tra i tribunali e le carceri, e che hanno una sinistra celebrità [...] dove sono misteriosi, ingegnosi, complicati nascondigli per gli oggetti rubati; dove talora per gli innumerevoli bugigattoli, trabocchetti, pozzi asciutti, andirivieni, pei camini in disuso, per le segrete e non interrotte comunicazioni tra i caseggiati di varie strade, si rintana, si rincattuccia, scappa un reo che va impunito ${ }^{21}$.

Esisteva del resto una forte solidarietà fra gli abitanti di questa zona così socialmente connotata e relativamente omogenea, anche contro i rappresentanti della legge:

[...] un certo numero di guardie andava, non è molto, a Malborghetto, per arrestare G.D., notissimo pregiudicato. Ma sorge subito la ribellione. Il G.D. è messo in una carrozza, è assicurato, come dicono gli agenti nel loro gergo; quando a un segno di convenzione scaturiscono dalle stalle [...] o da altri spechi i più famigerati malandrini: danno piglio a sassi, a strumenti da lavoro; fermano a forza la vettura: fanno scappare il G, e le guardie sono ferite ${ }^{22}$.

Le cronache narrano di molti casi in cui ladri o malviventi arrestati cercavano di resistere gridando e facendo appello alla popolazione, che spesso manifestava o quanto meno inveiva contro gli agenti.

\section{Criminali o sovversivi?}

In realtà le cronache giornalistiche, e alcuni degli scrittori che più avevano indagato i caratteri della "Firenze sotterranea", come Jarro, ci offrono un quado a fosche tinte di San Frediano, come il "quartiere de' ladri, de' malviventi" che non è evidentemente privo di riscontri, se non altro dalla altissima incidenza statistica degli ammoniti sorvegliati e pregiudicati, ma che appare piuttosto parziale, e motivato anche dal forte interesse delle classi dirigenti fiorentine a realizzare un'altra operazione urbanistica come quella del Ghetto, che avrebbe permesso un fortissimo incremento dei valori immobiliari in tutta la zona che andava dal centro alla zona altrettanto prestigiosa ed elegante del viale de' Colli.

${ }^{21}$ Ibidem, pp. 28, 41.

22 Ibidem, p. 67. 
In realtà San Frediano non era un gruppo di isolati circoscritto, e tutto uniformemente degradato. Era un rione popolare, molto vasto, al centro del popoloso e grande quartiere di Santo Spirito, da solo quasi un quarto di tutta la popolazione della città. Anche i sostenitori del carattere delinquenziale del quartiere e del risanamento radicale dovevano riconoscere che non vi erano solo delinquenti e sovversivi; accanto ad essi

Ci sono pure centinaia di poverissimi mestieranti, gente che si serba incontaminata al contatto più pestilenziale [...] una piccola popolazione, caduta all'imo di ogni miseria, quasi tutta malata, intristita, però non guasta, vive accanto all'altra, composta de' più fieri ribaldi ${ }^{23}$.

Naturalmente questo modo di vedere, per cui esistevano due San Frediano l'una bianca, l'altra nera, l'una corrotta e perduta, l'altra ancora incontaminata, rispecchiava, più che la realtà, le paure e le speranze della borghesia fiorentina. Come dovevano riconoscere le stesse cronache de «La Nazione», vi erano stati degli episodi in cui tutta la popolazione del quartiere era insorta compatta, senza distinzioni anche per motivi in cui non era in gioco una solidarietà fra malviventi, bensì qualcosa di piuttosto diverso. Un episodio avvenne alla vigilia della grande guerra, nei mesi in cui Firenze era uno dei centri più vivi dell'interventismo borghese, dell'intellettualità nazionalista $\mathrm{e}$ democratica. San Frediano era il quartiere che finì per interpretare il centro dell'opposizione popolare alla guerra, il pacifismo endemico e profondo della popolazione povera. Nel novembre 1914, gli studenti di un liceo cittadino ebbero l'idea, per sottolineare il loro patriottismo, di recarsi in gita a Bellosguardo, attraversando San Frediano al canto di inni patriottici e di evviva alla guerra. Come disse il Prefetto nel suo rapporto,

In tali condizioni di ambiente e in un quartiere popolare come quello di San Frediano dove abbondano i sovversivi e si annidano i peggiori elementi della malavita, centoventi studenti del Liceo Dante che si recavano ai Colli di Bellosguardo per una passeggiata scolastica, percorsero in colonna le vie, cantando inni patriottici ed emettendo qualche grido di «evviva la guerra» [...] male scelto

${ }^{23}$ Ibidem, pp. 35, 37. 
l'itinerario per uscire dalla città, peggior consiglio fu quello di ritornare per la stessa via...24

Infatti, al ritorno gli studenti trovarono ad attenderli, sempre usando le parole del prefetto, una "folla di popolo" composta insieme da "giovinastri" e da "sovversivi", che

si scagliarono contro gli studenti. Questi fecero atto di reagire, e furono allora colpiti con sassi e bastoni con maggior violenza. Il pronto intervento della forza pubblica evitò gravi conseguenze, perché peggio poteva accadere $[\ldots]^{25}$.

Le cronache narrano nei mesi seguenti di veri e propri scontri fra la folla dei manifestanti pacifisti che dopo aver manifestato in piazza de' Nerli si dirigevano verso il centro della città, e la polizia che cercava di fermarli sui ponti, che costituivano il passaggio obbligato e facilmente controllabile, con esiti alterni. Il 13 maggio ad esempio, nel pieno del "maggio radioso" delle manifestazioni interventiste in tutta Italia, per contrastare una manifestazione interventista prevista nel centro di Firenze, un folto gruppo di manifestanti «capitanati da un tale che portava legato in cima ad una pertica un drappo rosso, discendevano da San Frediano verso il centro della città. [Subito..] carabinieri e guardie corsero a quella volta» ed evidentemente proprio sul ponte avvenne lo scontro, dato che, come affermava il Prefetto, «Il Tenente Spanò impiegò una colluttazione col vessilllifero di San Frediano, e l'asta della improvvisata bandiera cadde nel fiume» ${ }^{26}$.

Si trattava del resto solo di un anticipo di quello che sarebbe successo nel 1921, quando il segretario della federazione comunista fiorentina, Spartaco Lavagnini, fu ucciso mentre era seduto alla sua scrivania da una squadra fascista penetrata nei locali di via Taddea, una delle vie popolari rimaste nel quartiere di San Lorenzo. La cintura rossa dei comuni attorno a Firenze insorse. San Frediano e Santo Spirito insorsero per tre giorni fino a che le autoblindo dell'esercito non soffocarono la rivolta; e in quel

24 ACS, MI, DGPS, A5G, b. 18B, rapporto prefettizio del 11 dicembre 1914, cit. in TOMASSINI, Luigi, Associazionismo operaio a Firenze fra '8oo e '9oo. La società di mutuo soccorso di Rifredi (1882-1922), Firenze, Leo S. Olschki Editore, 1984, p. 284.

25 Sull'episodio, cfr. CARETTI, Stefano, «Firenze nei mesi della neutralità», in Rassegna Storica Toscana, XXIII, 1/1977, pp. 67-100. La citazione in ACS, MI, DGPS, A5G, b. 18B, rapporto prefettizio del 11 dicembre 1914, cit. in TOMASSINI, Luigi, Associazionismo operaio a Firenze fra '8oo e '9oo. La società di mutuo soccorso di Rifredi (1882-1922), Firenze, Leo S. Olschki Editore, 1984, pp. 284; sull'episodio si veda anche CERASI, Laura, Democrazia, patriottismo, politica di massa. La massoneria in età giolittiana, in CONTI, Fulvio (a cura di), La massoneria a Firenze. Dall'età dei Lumi al secondo Novecento, Bologna, Il Mulino, 2007, pp. 304-305.

${ }_{26}$ ACS, MI, DGPS, A5G, b. 18B, rapporto prefettizio del 15 maggio 2015, cit. in TOMASSINI, Luigi, op. cit., p. 285. 
quadro si svolsero episodi come quello dell'uccisione di Giovanni Berta, gettato giù, secondo il martirologio fascista, da uno dei ponti che separavano il quartiere dal centro della città.

Ma anche questa rappresentazione del quartiere come una zona schiettamente popolare e politicamente compatta è altrettanto parziale di quella di Jarro o di Yorik. Al di là di questi episodi eclatanti, in cui si svela il volto di una compatta rivolta contro l'autorità, la realtà del quartiere quotidianamente aveva il volto di una microcriminalità diffusa e endemica, che si ritrova sì su «La Nazione» e sugli altri giornali cittadini, non nei pezzi di colore suggestivi ma soggettivi di Jarro e degli altri scrittori e giornalisti, bensì nella minuta e precisa cronaca nera.

\section{Piccoli delinquenti e lavoratori poveri}

Da un breve sondaggio che ho compiuto, la cronaca nera quotidiana, quella dei piccoli furtarelli, delle risse, delle piccole violenze, mette in crisi innanzitutto la netta distinzione fra delinquenti abituali e professionali, e lavoratori poveri ${ }^{27}$.

Ad esempio, in occasione di un clamoroso «furto di gioielli avvenuto nell'abitazione della signora Eugenia Ganusci ved. Pratesi, in Via S. Spirito 3», nel tratteggiare «gli autori del furto», il cronista li qualificava come «Giuseppe Innocenti, detto il Moro ladro di 35 anni carbonaio; Silvio Papini detto Burro, di 43 anni, intagliatore; Guido Percoveri, di 34 anni, ebanista disoccupato».

In altri casi erano donne, donne di servizio o donne senza condizione professionale certa, che compivano furtarelli di lieve entità, ma sempre registrati dalle cronache e puniti con severità:

La signora Mary S. Stressard, dimorante in Borgo San Frediano al n. 10, denunziava che una persona ben pratica della sua casa, le aveva involate alcune pezze di stoffa. Iniziate le relative indagini dall'ispettore Setti, questi potè stabilire che autrice del furto, era Gaetana Gallotti [...]. Dichiarata in arresto [...] fu associata alle carceri di Santa Verdiana. In una stanza in Via Maggio al n. 20 tenuta in affitto dalla Gallotti, fu sequestrata una pezza di stoffa, riconosciuta di proprietà della signora Stressard ${ }^{2}$.

\footnotetext{
${ }_{27}^{27}$ Prendendo in esame sistematicamente la cronaca nera della «Nazione» dell'estate 1898, ed enucleando gli episodi relativi a San Frediano.

${ }^{28}$ La Nazione, 20 luglio 1898. Tutte le pagine citate de «La Nazione» citate nell'articolo provengono dalla sezione Cronaca di Firenze.
} 
Per restare nel campo dei furti di stoffa, una tipologia piuttosto diffusa a quanto pare all'epoca,

il merciaio Moisè Soschiso, dimorante il via del Piaggione, mentre passava per via dello Sprone conducendo a mano il proprio barroccio con sopra pezze di stoffa e di cambrai ebbe a fermarsi per servire un avventore. Una donna di pessimi costumi, dimorante in quella via, approfittando del momento, rubò destramente di sul barroccio una pezza di cambrai e fuggi via ${ }^{29}$.

Quello dei furti ai commercianti esterni che dalla via Romana o dal Poggio Imperiale entravano nel quartiere dirigendosi verso il centro, sembra fosse una tipologia abbastanza frequente, cosa che ci permette di immaginare una piccola delinquenza di passo non proprio professionale. Questo non esclude naturalmente che in altri casi, non fossero all'opera ladri di mestiere. Ci sono testimonianze da cui emerge che a San Frediano il mestiere di ladro poteva essere una qualifica professionale vera e propria allo stesso modo di un lavoro artigiano.

Accanto alla piccola delinquenza di passo non proprio professionale, assai frequenti erano anche i furti di ladri professionisti che si introducevano di notte all'interno delle botteghe:

La guardia di notte, Vittorio Marini, perlustrando stanotte Borgo San Frediano, vide dalla merceria di Luigi Anichini posta al n. 83, fuggire alcuni individui in direzione della Porta. Egli pose senz'altro a inseguirli e per richiamare l'attenzione di qualche pattuglia e di agenti della pubblica forza, sparò due colpi di revolver in $\operatorname{aria}^{30}$.

Scene simili erano all'ordine del giorno in San Frediano: ladri colti sul fatto che fuggivano, pregiudicati che sorpresi dalla polizia saltavano dalla finestra delle loro abitazioni, inseguimenti, colpi di rivoltella e così via.

Emerge anche una criminalità associata a vicende passionali o a liti familiari. Un grave fatto di cronaca che vide un omicidio compiuto da un militare, non fiorentino, in servizio in una caserma ai limiti del quartiere, ai danni di una giovane lavoratrice, occupò le pagine dei quotidiani cittadini per più di un anno3¹.

\footnotetext{
29 La Nazione, 13 luglio 1898.

3o La Nazione, 8 agosto 1898.

${ }^{31}$ Vedi «La Nazione» per tutto il 1898. 
Oltre agli episodi di micro-criminalità fatta di scippi ad opera anche di «individui decentemente vestiti», risse, accoltellamenti quotidiani, ma anche omicidi non necessariamente passionali, legati ai «misteri del quartiere», altre attività illecite proliferavano nella zona. Accanto alle bische clandestine in cui la polizia faceva periodicamente irruzione, nel maggio del 1898 «la morte improvvisa della giovane Costanza Cacciapelli [...] destò de' sospetti che furono riferiti all'Autorità giudiziaria». La perizia medica stabilì che la fine repentina della sciagurata era stata la conseguenza di manovre abortive. Le indagini condotte nei giorni successivi dalla polizia, accertarono che il delitto fu commesso con l'aiuto di certa Caterina Magnese, abitante in Via Romana al n. $45^{32}$. Poche settimane dopo, nella stessa zona, «in Via del Poggio Imperiale», proprio nei pressi di Porta Romana, «presso la Villa Farina, fu trovata una cassetta contenente un feto di sesso mascolino»33.

Occorrerebbe un'analisi molto più sistematica e ampia di quella che ho potuto compiere, ma da questo primo sondaggio si ricava l'impressione che Santo Spirito e San Frediano ricorrano effettivamente con una presenza chiaramente superiore a quella di altri quartieri nella cronaca nera, che la gran parte di questa delinquenza sia bagatellare, e coinvolga non solo malviventi professionali, ma anche delinquenti occasionali, spinti spesso dalla povertà estrema e dal disagio sociale.

\section{Una fame nera: le indagini sociali e le fonti mediche e giudiziarie}

Il disagio e il degrado colpivano nel quartiere altresì le condizioni elementari di vita, a cominciare dall'alimentazione, anche quelle parti di popolazione, come i bambini, che erano più deboli e bisognosi di protezione. Alla fine del secolo, Gattesco Gatteschi così descriveva la condizione fisica, oltre che morale, dei piccoli:

I bambini poveri a Firenze, diciamolo pur francamente, non sono di fibra molto robusta: i cerchi azzurrognoli dell'occhiaia fanno quasi sempre da livida cornice agli occhietti intelligenti e vivaci; e i corpiciattoli panciuti riposano gravi sul tenue piedistallo delle gambette sottili34.

Secondo altri autorevoli autori, negli "abituri" poveri dei "camaldoli" di San Frediano:

${ }^{32}$ La Nazione, 8 luglio 1898.

33 La Nazione, 28 luglio 1898.

34 Firenze d’oggi, Firenze, Tip. Ariani, 1896, p. 42. 
brulica una famiglia che attende sempre il domani con disperazione; i suoi figli abbandonano di buon mattino la casipola dove condivisero anguste ed umide pareti, poca ed impura l'aria, scarsi cenci per letto; essi salutano il giorno con una visita alla rivendita di liquori ed il primo ristoro è una tristissima acquavite che chiamano zozza, e quando l'economia lo permetta, l'accompagnano ad un pane di miglio, $o$ ad altro genere di pasta35.

Questa situazione all'inizio del secolo provocò un ritorno di attenzione fortissimo verso San Frediano non solo da parte di scrittori o giornalisti, ma anche da parte di studiosi, di enti e amministrazioni pubbliche a cominciare dal comune, che specialmente nel periodo dell'amministrazione popolare promosse una serie d'indagini statistiche che misero in luce come tutta una serie di indicatori fossero tali da dimostrare una situazione di disagio speciale nel quartiere di Santo Spirito e nel suo cuore più oscuro, cioè a San Frediano ${ }^{36}$.

Come mostra la tabella seguente, il quartiere di Santo Spirito, entro il quale era completamente compreso il rione di San Frediano, che ne costituiva la parte più popolare e disagiata, era nettamente sfavorito, rispetto alla media della città, da tutta una serie di indicatori, in particolare dai vari indicatori di mortalità (generale, infantile, per tubercolosi, all'epoca malattia sociale particolarmente diffusa a Firenze), e di sovraffollamento nelle abitazioni.

Tabella 1: Indici di disagio sociale nel quartiere di Santo Spirito37

\begin{tabular}{|c|c|c|c|c|c|c|c|}
\hline & $\begin{array}{c}\text { Densità } \\
\text { (abitanti/ettaro) }\end{array}$ & $\begin{array}{c}\text { Mortalità generale } \\
\text { su 10.00o abitanti }\end{array}$ & $\begin{array}{c}\text { Mortalità } \\
\text { per TBC }\end{array}$ & $\begin{array}{c}\text { Mortalità } \\
\text { infantile (morti } \\
\text { fino a 12 mesi su } \\
\text { 10o nati) }\end{array}$ & $\begin{array}{c}\text { Poveri } \\
\text { su 10o sposi }\end{array}$ & $\begin{array}{c}\text { Sovraffollati } \\
\text { su 100 abitanti }\end{array}$ & $\begin{array}{c}\text { Mobilità } \\
\text { infantile }\end{array}$ \\
\hline $\begin{array}{c}\text { Quartiere di } \\
\text { Santo Spirito }\end{array}$ & $\mathbf{3 9 2}$ & $\mathbf{2 2 , 4 0}$ & $\mathbf{3 9 , 7 0}$ & $\mathbf{1 6 , 2 0}$ & $\mathbf{4 1 , 5 0}$ & $\mathbf{1 8}$ & $\mathbf{2 , 5 5}$ \\
\hline $\begin{array}{c}\text { Media di tutti i } \\
\text { quartieri di } \\
\text { Firenze }\end{array}$ & $\mathbf{1 5 3}$ & $\mathbf{1 7 , 6 7}$ & $\mathbf{2 9 , 4 8}$ & $\mathbf{1 0 , 5 9}$ & $\mathbf{3 9 , 1 6}$ & $\mathbf{9 , 9 3}$ & $\mathbf{2 , 3 8}$ \\
\hline
\end{tabular}

35 WOOLF Stuart Joseph, Come e che cosa mangiavano i fiorentini cent'anni fa?, in «Ricerche storiche», XXVIII, 3/1998, pp. 483-506, p. 496.

${ }^{36} \mathrm{Cfr}$. in particolare COMUNE DI FIRENZE, Inchiesta sulle abitazioni popolari (ottobre 1907), Firenze, Tip. Chiari, 1909; UNIONE FIORENTINA PER IL MIGLIORAMENTO E RISORGIMENTO DEL QUARTIERE D'OLTRARNO, Origine, scopo e azione dell'Unione fiorentina per il miglioramento e risorgimento del quartiere d'Oltrarno, Firenze, Tip. Rinaldi e Ciani, 1913; COMUNE DI FIRENZE, Le abitazione e i redditi delle classi popolari fiorentine nel 1914, Firenze, Tip. Ariani, 1914.

37 La tabella è tratta da TOMASSINI, Luigi, op. cit., pp. 212-213. 
Non ci possiamo soffermare in questa sede su questo tipo d'indagini, su cui esistono peraltro già alcuni studi approfonditi38; ma ci interessa stabilire che anche in virtù di questa attività di inchiesta e di indagine sociale presero sempre maggior vigore i progetti del "risanamento" del quartiere. Un risanamento che doveva infine eliminare il cuore nero di Firenze e restituirlo alla luce; come notava un osservatore:

«Date aria, date luce! Il che vuol dire: date salute, moralità!»39.

\section{Progetti e fallimenti del "risanamento"}

La campagna di denuncia del degrado della zona della città di Santo Spirito e Oltrarno, e soprattutto del suo "cuore nero" nelle zone interne del quartiere, il borgo di San Frediano e il Canto dei quattro Leoni, doveva quindi portare a una serie di iniziative per correggere questa situazione e nel contempo, così come era avvenuto nel caso del Ghetto, realizzare anche un miglioramento dei valori fondiari non indifferente.

I progetti di risanamento quindi furono continui e molto precoci.

Già nel 1869 un gruppo di proprietari ed inquilini aveva denunciato lo stato della zona, ingombra di «abitazioni indecenti e asilo in gran parte di gente trista e svergognata»40; sei anni più tardi gli stessi cittadini avevano premuto sul sindaco perché «nello interesse del pubblico decoro e del privato vantaggio voglia considerarsi come potesse contemporaneamente anche a questa importante porzione della nostra città darsi assestamento conveniente e aspetto condegno del generale suo ed aumentato lindore» ${ }^{4}$. Col passare degli anni queste proteste e richieste si tradussero in ipotesi di radicale risanamento, che giunsero fino a prevedere la distruzione di larga parte del quartiere, come nel progetto di L. Rimbotti del 1887, che prevedeva di abbattere «quel

\footnotetext{
${ }^{8} \mathrm{Si}$ veda fra gli altri GIUNTINI, Andrea, «Tutto alla fogna. Igiene e infrastrutture urbane a Firenze fra Ottocento e Novecento», in Ricerche Storiche 3/1998, pp. 508-545, pp. 510-511; BRUNI, Domenico Maria, VISCIOLA, Simone (a cura di), Il Comune popolare e l'igiene sociale a Firenze, Manduria, Lacaita, 2003; cfr. inoltre il recente saggio di DEGL'INNOCENTI, Maurizio, Scienza, amministrazione e politica nel governo della città. L'esperienza fiorentina, in ID. (a cura di), La cultura delle riforme tra Ottocento e Novecento, Manduria, Lacaita, 2003, pp. 23-57. Si veda più in generale sulle politiche sociali dei "popolari" al governo PICCIOLI, Lorenzo, I "Popolari" a Palazzo Vecchio, amministrazione politica e lotte sociali a Firenze dal 1907 al 1910, Firenze, Olschki, 1989. Peraltro tale politica si allineava ad una tendenza in atto in molte citta europee, si veda per il caso francese MURARD, Lion, ZYLBERMAN, Patrick, L'higiène dans la République. La santé publique en France ou l'utopie contrariée 1870-1918, Paris, Fayard, 1996.

39 JARRO, Firenze sotterranea cit., p. 36.

40 FANTOZZI MICALI, Osanna, La città desiderata, Firenze come avrebbe potuto essere: progetti dall'Ottocento alla seconda guerra mondiale, Firenze, Alinea, 1992, p. 178.

${ }^{41}$ Ibidem.
} 
più che sarà necessario» costruendo larghe strade e rendendo il quartiere «arieggiato» con la costruzione di piccoli fabbricati di tre piani. Durante l'età giolittiana si costituì una «Unione fiorentina per il miglioramento e il risorgimento del quartiere d'Oltrarno» che premette sulle autorità e sull'opinione pubblica per ottenere almeno il risanamento delle zone più degradate.

Alla vigilia della grande guerra questi progetti trovarono una sintesi ad opera dell'ing. Giovanni Bellincioni, il quale aveva titolo per parlarne in quanto copriva la carica di assessore ai lavori pubblici del comune di Firenze. Bellincioni conduceva una analisi accurata e puntuale di tutte le forme di disagio e di degrado che si riscontravano in San Frediano, valendosi ampiamente dell'ottima opera di Ugo Giusti e dell'ufficio di statistica del Comune; ma poi ammetteva esplicitamente che lo scopo che si poneva l'amministrazione di cui faceva parte (di orientamento moderato) non era quello di risolvere il problema igienico di quella zona della città: si trattava non solo e non tanto di risanare gli edifici, «ma anche e più che tutto renderli adatti a famiglie di condizione sociale più elevata» 42 .

Questo obiettivo faceva sì che gli interventi mirati a risanare le parti più degradate non fossero efficaci allo scopo: infatti si sarebbero potuti evitare alcuni dei disagi più drammatici, ma sarebbe rimasto irrisolto il problema di fondo che orientava tutti i progetti moderati di riqualificazione del centro urbano, tutti i tentativi di ridisegnarlo alla luce di nuovi criteri di rispettabilità, funzionalità, decoro. Come, infatti, convincere «quella famiglia di condizione civile per quanto finanziariamente modesta che si adatterà ad abitare lo stabile trasformato, quando esso continuerà a trovarsi ai lati e in faccia circondato da altri edifici che dieno ricovero a gente sudicia e di bassa moralità?»43.

L'unica soluzione era quella di risanare l'intera zona, di espellere gli abitanti poveri e non rispettabili, e per questo il Bellincioni preparò un piano imponente, che doveva offrire la possibilità di costruzione di 6.000 stanze e favorire anche lo sviluppo delle zone retrostanti.

Le pittoresche colline di Bellosguardo, liberate dal contatto dannoso dell'attuale misero quartiere di S. Frediano, poste con un bel viale in contatto col nuovo elegante quartiere, si popoleranno di nuove ville e di moderni grandiosi villini, e

${ }^{2}$ BELLINCIONI, Giovanni, op. cit., p. 15.

43 Ibidem, p. 16. 
renderanno ben giustificata quella prosecuzione del viale dei colli che era nella mente dell'architetto Poggi44.

Si trattava di un progetto che si poneva un obiettivo sensibilmente innovativo. Infatti, lo sviluppo precedente e contemporaneo della città aveva visto due tipologie prevalenti: quella dei palazzi estremamente lussuosi o delle zone di rappresentanza ricostruite nel vecchio centro, e quello delle casette o villette da 4 a 10 stanze nelle zone immediatamente periferiche. Vi era un mercato potenziale molto ampio per una tipologia edilizia ed urbanistica nuova e più moderna, quella dei grandi palazzi "signorili" destinati ad inquilini delle classi medio alte, una tipologia che era chiaramente insufficiente nel capoluogo secondo il Bellincioni:

I nuovi quartieri percorsi da linee tranviarie a doppio binario, bene areati e illuminati, con strade ampie, fiancheggiate da palazzi veramente moderni costruiti con tutti i perfezionamenti della moderna edilizia, verranno a colmare una lacuna assai sentita nell'edilizia fiorentina, quella consistente nella mancanza di palazzi moderni da inquilini nell'interno della città 45 .

Evidentemente si mirava solo e tanto al risanamento, ma alla riqualificazione di una parte importante della città:

\footnotetext{
Non si tarderà a riconoscere che l'obiettivo che io mi sono proposto non è stato solamente il risanamento di uno di quei quartieri che lo studio fatto ci ha dimostrato trovarsi nelle peggiori condizioni, ma bensì quello di trasformare quella parte della città in un quartiere moderno che della modernità abbia tutti i requisiti46.
}

Una operazione del genere avrebbe avuto senso solo se applicata integralmente; avrebbe trasformato l'Oltrarno, da quel dedalo strettamente intrecciato di vie, viuzze e palazzi nobiliari, in una zona residenziale moderna, elegante con strade larghissime e ampie piazze, collegata con un nuovo ponte al centro cittadino. Sotto questo aspetto valeva per l'assessore fiorentino il principio del «tanto peggio, tanto meglio»: gli interventi limitati di risanamento igienico avrebbero infatti ostacolato l'effettivo «risanamento». Come dichiarava egli stesso:

\footnotetext{
44 Ibidem, p. 25.

45 Ibidem, p. 24.

${ }^{46}$ Ibidem, p. 23.
} 
Sino ad un certo limite dobbiamo anzi temere dell'opera dei privati proprietari di questi modesti edifici, quando essa si sforzi di rabberciarli e renderli meno squallidi in quanto questi modestissimi miglioramenti precludono la via a miglioramenti radicali, o quantomeno li rendono più onerosi 47.

In queste parole si trovano forse le risposte agli interrogativi posti in precedenza. La classe dirigente fiorentina aveva nutrito propositi estremamente ambiziosi per lo sviluppo esteriore della città; non avrebbe perciò potuto ripiegare su soluzioni modeste e parziali. Per contro, nei quartieri popolari, probabilmente un fitto tessuto di attività produttive e commerciali che faticosamente tenevano il passo e resistevano nei modi che abbiamo visto alla pressione delle produzioni seriali, avrebbero contribuito a «rendere meno squallidi» quei quartieri, a farli ancora vitali, rendendo così più complicata e difatti rimandando sine die l'opera di risanamento radicale proposta dagli ingegneri sociali di primo Novecento.

47 Ibidem, p. 17. 


\section{* L'autore}

Anna Pellegrino, assegnista di ricerca Senior presso l'Università degli studi di Padova, è dottore di ricerca dell'Istituto Universitario Europeo (2004). Ha conseguito il DEA in Histoire et civilisation presso L’EHESS di Parigi (1999). Dal 2008 è chercheur associé al Conservatoire National des Arts et Métiers di Parigi, e dal 2010 all'Université Paris Diderot 7. Si occupa di storia delle culture del lavoro nella società industriale, di associazionismo operaio e popolare fra Otto e Novecento e di esposizioni universali fra XIX e XX secolo. Fra le sue pubblicazioni Macchine come fate. Gli operai italiani alle esposizioni universali 1851-1911, Milano, Guerini e Associati, 2011; La citta più artigiana d'Italia. Firenze 1861-1929, Milano, FrancoAngeli, 2012, e diversi saggi in riviste italiane e straniere.

URL: < http://www.studistorici.com/progett/autori/\#Pellegrino >

\section{Per citare questo articolo:}

PELLEGRINO, Anna, «Firenze noir. Criminalità e marginalità a Firenze tra Otto e Novecento», Diacronie. Studi di Storia Contemporanea : Le città di Babele, 29/3/2015,

URL:< http://www.studistorici.com/2015/3/29/pellegrino_numero_21/ >

\section{Diacronie Studi di Storia Contemporanea $\widehat{B}$ www.diacronie.it}

Risorsa digitale indipendente a carattere storiografico. Uscita trimestrale. redazione.diacronie@hotmail.it

Comitato di redazione: Jacopo Bassi - Luca Bufarale - Elisa Grandi - Deborah Paci - Fausto Pietrancosta - Matteo Tomasoni - Luca Zuccolo

Diritti: gli articoli di Diacronie. Studi di Storia Contemporanea sono pubblicati sotto licenza Creative Commons 2.5. Possono essere riprodotti a patto di non modificarne i contenuti e di non usarli per fini commerciali. La citazione di estratti è comunque sempre autorizzata, nei limiti previsti dalla legge. 